\title{
Low Power Complex Multiplication using Pre-computation Technique For FFT Algorithm in Wearable ECG Gadgets
}

\author{
Pankaj U Joshi ${ }^{1}$, and Vipul S Lande ${ }^{* 2}$ \\ ${ }^{1}$ Department of Electronics Engineering, \\ ${ }^{2}$ Department of Electronics \& Communication Engineering, \\ Shri Ramdeobaba College of Engineering and Management Nagpur, \\ Maharashtra, India
}

\section{ABSTRACT}

The focus on wearable devices for biomedical applications is gaining a lot of research and market interest. Heart diseases remain by far the main cause of death and a challenging problem for biomedical engineers to monitor and analyze. Electrocardiography (ECG) is an essential practice in heart medicine. However, wearable ECG gadgets for real time analysis still faces computational challenges, especially when multiple lead signals are to be analyzed in parallel, in real time, and under increasing sampling frequencies and battery operated gadgets. Another challenge is the computation of Fast Fourier Transform (FFT) on huge amounts of data that may grow to days of recordings. In this research we present the comparative study of FFT calculation best suitable for power optimized applications in the biomedical field and exclusive performance enhancement by reducing dynamic power consumption. ECG application specific to FFT calculation to the final hardware-software( $\mathrm{HW} / \mathrm{SW}$ ) architecture is the focus of this paper.

\section{KEY WORDS: WEARABLE ECG DEVICE, FFT COMPUTATION SCHEME, POWER CONSUMPTION IN COMPLEX FFT, ELECTROCARDIOGRAM ALGORITHMS.}

\section{INTRODUCTION}

Cardiovascular diseases (CVDs) have been on top behind the cause of deaths globally. According to the World Health Organization (WHO) report an estimated 17.3 million people died from CVDs in 2008, representing $30 \%$ of all global deaths. Of these deaths, an estimated 7.3 million were due to coronary heart disease and 6.2 million were due to stroke. WHO has predicted, by 2030, almost 23.6 million people will die from CVDs, mainly from heart disease and stroke (https://www.who.int/

\section{ARTICLE INFORMATION}

*Corresponding Author: joshipu@rknec.edu

Received 19th Oct 2020 Accepted after revision 24th Dec 2020

Print ISSN: 0974-6455 Online ISSN: 2321-4007 CODEN: BBRCBA

Thomson Reuters ISI Web of Science Clarivate Analytics USA and Crossref Indexed Journal

\section{Clarivate
Analytics}

NAAS Journal Score 2020 (4.31)

A Society of Science and Nature Publication,

Bhopal India 2020. All rights reserved.

Online Contents Available at: http//www.bbrc.in/

Doi: http://dx.doi.org/10.21786/bbrc/13.14/93 cardiovascular_diseases/about_cvd/en/). Due to rapid increase in cardiac diseases, the rate of hospitalization has drastically increased followed by periodic cardiac examination. This have demanded more efficient methods of cardiac diagnosis and desired to meet the great demand on heart examinations (Fuster V. 1999).

However, the traditional biomedical equipment for heartbeat sensing and monitoring lacks the ability of providing large-scale analysis and remote, real time computation at the patient's location. With the advancement of sensing, processing and communicating technology in the field of biomedical have encouraged the researchers to focus on such issues. This demand has given opportunities to design and modify biomedical equipments as modular and portable gadgets to facilitates the remote diagnosis. Electrocardiography (ECG) is a popular measure to quickly investigate the CVDs. It is desired that such continuous monitoring ECG gadgets should not be power hungry devices (Harland et al. 2002 and 2003). Therefore many power optimized solutions for 
devices are proposed in past. This work is focused on a new approach of power optimization in FFT estimation for ECG monitoring. The table I shows the summary of multiplication operations in N point FFT algorithms. It can be seen that multiplication operations are very large in the number for high resolution and practical FFT applications.

Table 1. Comparison of FFT for Real and Complex value signal(RFFT stands for Real FFT and CFFT stands for Complex FFT)

\begin{tabular}{|c|c|c|c|c|c|c|}
\hline \multirow{2}{*}{$\mathrm{N}$} & \multirow{2}{*}{ Regular CFFT } & Optimized CFFT & RFFT & $\begin{array}{c}\text { Optimized CFFT } \\
\text { Vs } \\
\text { Regular CFFT }\end{array}$ & $\begin{array}{c}\text { RFFT } \\
\text { Vs } \\
\text { Regular CFFT }\end{array}$ & $\begin{array}{c}\text { RFFT } \\
\text { Vs } \\
\text { Optimized } \\
\text { CFFT }\end{array}$ \\
\hline 1024 & 20480 & 13824 & 7172 & 32.5000 & 64.9805 & 48.11921296 \\
\hline 2048 & 45056 & 30720 & 16388 & 31.8182 & 63.6275 & 46.65364583 \\
\hline 4096 & 98304 & 67584 & 36868 & 31.2500 & 62.4959 & 45.44862689 \\
\hline 8192 & 212992 & 147456 & 81924 & 30.7692 & 61.5366 & 44.44173177 \\
\hline 16384 & 458752 & 319488 & 180228 & 30.3571 & 60.7134 & 43.58849159 \\
\hline 32768 & 983040 & 688128 & 393220 & 30.0000 & 59.9996 & 42.85656157 \\
\hline
\end{tabular}

FFT algorithm involves four multiplication operations for a complex multiplier. Mathematically these four multiplications can be converted into three multiplication operations to reduce the computational time and power. This conversion involves pre-computations, leading to change in the signal patterns, entering to the FFT block. We explore the possibility of best pre-computation technique to reduce the dynamic power consumption.

II. Background And Related Work: Fast Fourier Transform (FFT) architecture has a great significance in the world of signal processing. The literature has witnessed many optimization techniques to improve its performance at algorithmic levels, architectural level, system level and using signal properties. This section discuss about some milestone techniques related to FFT and its architectural optimization. The recent contribution by (S. M. Noor et al. 2019) proposed a lookup memory to provide the most frequent multiplication result for the most frequent multiplication operands. It uses the statistical approach to identify the most frequent multiplication operands for the ECG signal as input to the FFT block. (R. Neuenfeld et al. 2016) propose the optimization of butterfly for the power optimization of FFT algorithm, along with the reordering technique to avoid one adder and one subtractor operation in the butterfly unit. (Chu Yu and Mao-Hsu Yen 2015; W. Tsai et al. 2019) Utilizes the properties of twiddle factors and constant multiplication techniques to meet the low area and power requirements. The complex multiplication involving four multiplications is reduced to three multiplications has been reported in the past literature. However there are eight different techniques to represent this conversion. These techniques are not explored with respect to their dynamic power consumptions. We explore the scope of this finding for ECG signal as test input.

III. Mathematical Variations For Converting Four Multipliers To Three Multipliers: A DFT of an N point input signal is given by Equation (1) shows the mathematical formulation of the complex multiplier.

$$
(A+j B) \times(C+j D)=(A C-B D)+j(B C+A D-
$$

Figure 1: Butterfly as Processing element.

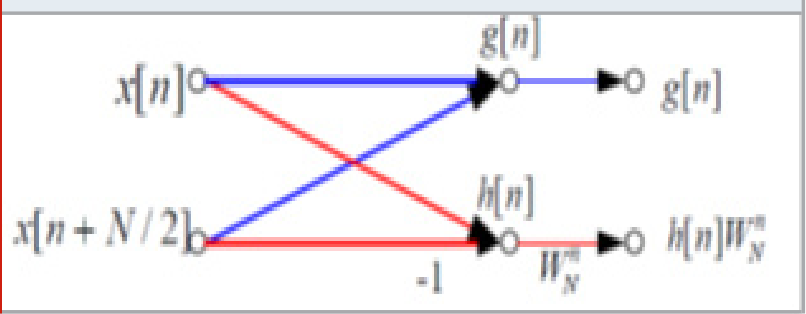

Right hand side of the Equation (2) shows the four real multiplications as $\mathrm{AC}, \mathrm{BD}, \mathrm{BC}, \mathrm{AD}$. AC and $\mathrm{BD}$ represent the real part while $\mathrm{BC}$ and $\mathrm{AD}$ represents the imaginary part of the complex multiplier. Mathematically this complex multiplier can be expressed using only three multiplications by manipulating the equation (2).

\section{Proposed Work: Precomputation For Complex Multiplication}

This section discusses about the 1024 point FFT computation using different complex multipliers schemes. FFT has a butterfly section, using which iteratively; N point FFT is generally computed. A butterfly section involves four multiplication operations. These four multiplications can be converted into three multiplications using eight different mathematical schemes given below. We have derived all the possible variants for converting the four multipliers of complex multiplication into three multiplications.

$\begin{array}{llll}\text { Scheme I: } & \text { Real Part: } A C-B D & \ldots & \text { (I.I) } \\ & \text { Imaginary Part: }(A+B) \times(C+D)-A C-B D & \ldots & \text { (I.I) } \\ \text { Scheme II: } & \text { Real Part: }(A+B)(C+D)-B C+A D & \ldots & \text { (I.I) } \\ & \text { Imaginary Part: } B C+A D & \ldots . & \text { (I.I) } \\ \text { Scheme III: } & \text { Real Part: } A(C+D)-D(A+B) & \ldots . & \text { (III.I) } \\ & \text { Imaginary Part: } D(A+B)+B(C-D) & \ldots . & \text { (III.II) } \\ \text { Scheme IV: } & \text { Real Part: } A(C+D)-D(A+B) & \ldots . & \text { (IV.I) } \\ & \text { Imaginary Part: } A(C+D)-C(A-B) & \ldots . & \text { (IV.II) } \\ \text { Scheme V: } & \text { Real Part: } B(C-D)+C(A-B) & \ldots . & \text { (V.I) } \\ & \text { Imaginary Part: } B(C-D)+D(B+A) & \ldots . & \text { (V.II) } \\ \text { Scheme VI: } & \text { Real Part: } D(A-B)+A(C-D) & \ldots . & \text { (VI.I) } \\ & \text { Imaginary Part: } B(C+D)+D(A-B) & \ldots . & \text { (VI.II) } \\ \text { Scheme VII: } & \text { Real Part: } C(A+B)-B(C+D) & \ldots . & \text { (VII.I) } \\ & \text { Imaginary Part: } C(A+B)+A(D-C) & \ldots . & \text { (VII.II) } \\ \text { Scheme VIII: } & \text { Real Part: } B(C-D)-C(B-A) & \ldots . & \text { (VIII.I) } \\ & \text { Imaginary Part: } C(B-A)+A(C+D) & \ldots . & \text { (VIII.I) }\end{array}$

It is to note that the input signal applied to an $\mathrm{N}$ point FFT block is passed through the pre-computation process. For example in a scheme I, $(\mathrm{A}+\mathrm{B})$ and $(\mathrm{C}+\mathrm{D})$ are precomputed. The figure 2 shows the section of MATLAB code utilizing the one of the pre-computation schemes in the complex multiplication of the butterfly. This work proposes to analyze dynamic power values in the complex multiplier due to the effect of pre-computations for the eight different schemes derived in equation I to VIII. 
Functional Verification of the 1024 point Radix2-DIT FFT algorithm is done using a ECG signal taken from the MIT BIH (https://archive.physionet.org/physiobank/database/ mitdb/) data base. Figure 3a shows the 1024 points of data extracted from the ECG signal. The signal is sampled by frequency $250 \mathrm{~Hz}$. The duration of data is $5.384 \mathrm{sec}$. The Figure $3 \mathrm{~b}$ shows the theoretical values of the FFT algorithm using direct FFT command of MATLAB. These samples are supplied to the FFT algorithm written in matlab for functional verification. Figure 4 shows the visual comparison of the magnitude Vs. frequency spectrum of FFT algorithm with eight different complex multiplication schemes. All the eight schemes show exactly same Magnitude Vs. frequency plot. Further, we have calculated the cross -correlation of the each scheme with the theoretical value of the FFT using direct command. All the schemes show the maxima on same index value as shown in the Figure 5. This confirms the functional integrity of all the eight schemes.

Power Performance observations: The dynamic power consumption of the N point FFT architecture is highly dependent on the bit patterns. The eight different schemes are responsible for generation of different bit patters. The Table I shows the dynamic power consumed by each multiplier of the scheme for 1024 point FFT calculations. The algorithm is implemented using a Microblaze soft core processer in a XilinxXC3S500E5FG320C for functional verification on FPGA platform. The ECG signal samples taken from MIT BIH library as test signal. The test signal going as input to the multiplication operation in each stage is captured using soft core processor. The captured signal is then passed to the multiplier using VHDL test bench for dynamic power measurement in Xilinx x-power utility.

Figure 2: Section of Matlab code with complex multiplication realized using three multipliers.

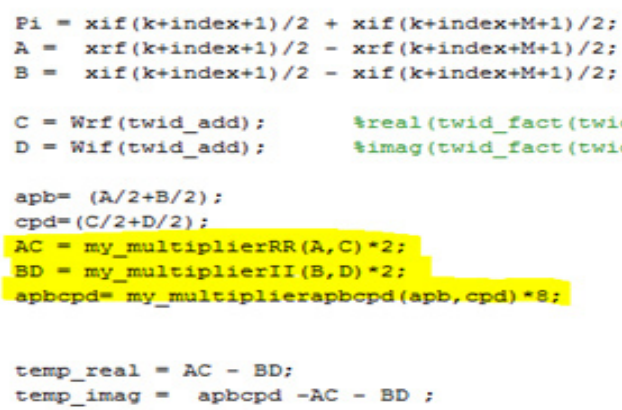

Experimental Results: It can be observed that the all the eight schemes show different total dynamic power consumption values. Some of the schemes have given a lower dynamic power every time even though a different ECG data set is applied. The pre-computations in each of the schemes generate different input bit patterns for $\mathrm{N}$ point FFT architecture. The different bit patterns, generated in each of the eight schemes, pass through $\mathrm{N}$ stages of the FFT architecture.
Figure 3: (a) Four cycles of ECG signal with $5.834 \mathrm{sec}$, duration N=1024 samples, Sampling frequency: $250 \mathrm{~Hz}$. (b) Theoretical Value of FFT of a ECG signal using FFT command.

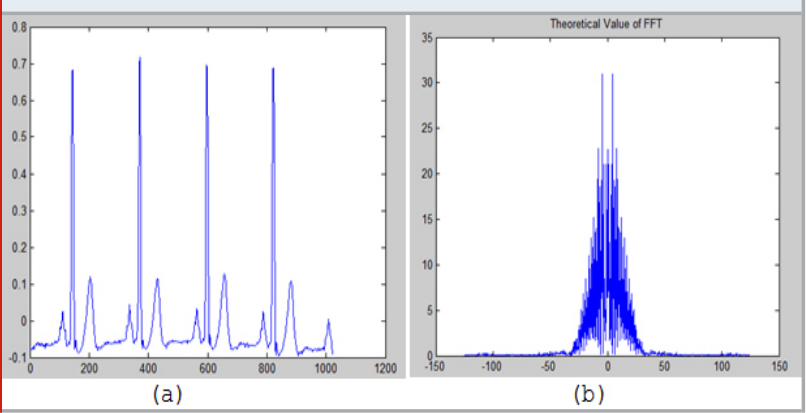

Figure 4: Magnitude Vs. Frequency Spectrum of the FFT algorithm using eight different complex multiplication schemes

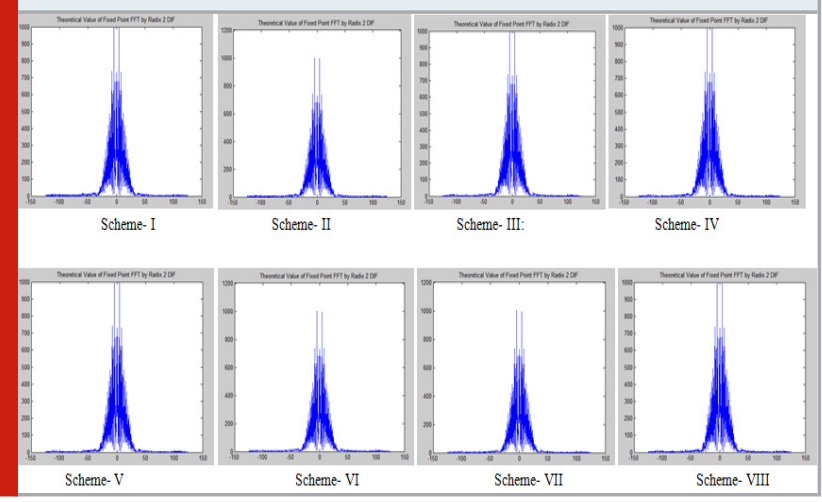

Figure 5: Index matching using Cross correlation results of each of the schemes with the theoretical values of the FFT

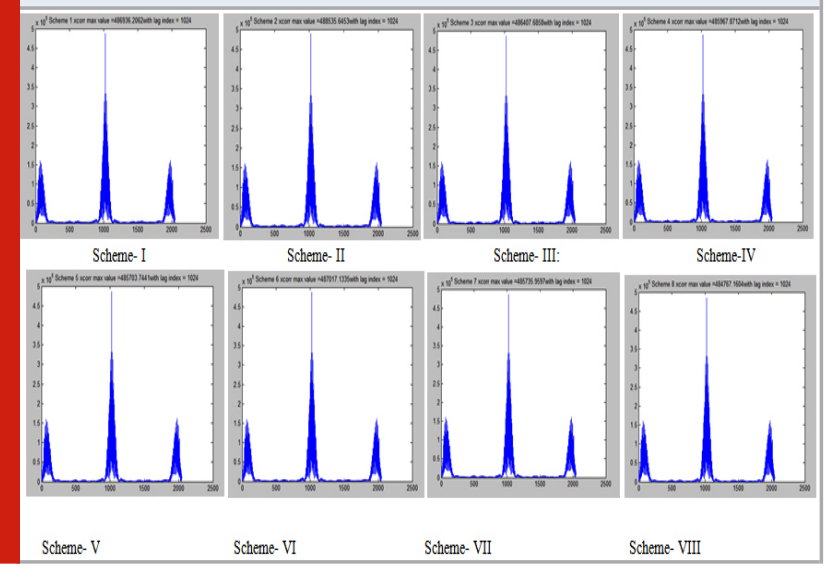

However, the result of the Nth stage of the FFT in terms of the frequency verses magnitude plot is same, even though the bit patterns are different at every stage. Therefore, intelligent selection of a low power complex multiplication scheme will make the overall design power efficient. 
Table 2. Dynamic power consumption in multiplier components of scheme I to VIII.

\begin{tabular}{|c|c|c|c|}
\hline Schemes & $\begin{array}{c}\text { Mathematical } \\
\text { Equation }\end{array}$ & $\begin{array}{l}\text { Dynamic power in multiplier } \\
\text { without power optimization }\end{array}$ & $\begin{array}{l}\text { Total Dynamic } \\
\text { Power }\end{array}$ \\
\hline I & $\begin{array}{c}\text { Real }=A C-B D ; \\
\operatorname{Img}=(A+B)(C+D)-A C-B D ;\end{array}$ & $\begin{array}{c}\mathrm{AC}=50 \\
\mathrm{BD}=38 \\
(\mathrm{~A}+\mathrm{B})(\mathrm{C}+\mathrm{D})=47\end{array}$ & $135 \mathrm{~mW}$ \\
\hline II & $\begin{array}{c}\text { Real: }(\mathrm{A}+\mathrm{B})(\mathrm{C}-\mathrm{D})-\mathrm{BC}+\mathrm{AD} \\
\text { Img: } \mathrm{BC}+\mathrm{AD}\end{array}$ & $\begin{array}{c}\mathrm{BC}=41 \\
\mathrm{AD}=48 \\
(\mathrm{~A}+\mathrm{B})(\mathrm{C}-\mathrm{D})=43\end{array}$ & $132 \mathrm{~mW}$ \\
\hline III & $\begin{aligned} \text { Real } & =A(C+D)-D(A+B) \\
I m g & =D(A+B)+B(C-D)\end{aligned}$ & $\begin{array}{l}\mathrm{B}(\mathrm{C}-\mathrm{D})=34 \\
\mathrm{~A}(\mathrm{C}+\mathrm{D})=48 \\
\mathrm{D}(\mathrm{A}+\mathrm{B})=41\end{array}$ & $123 \mathrm{~mW}$ \\
\hline IV & $\begin{array}{l}\text { Real }=A(C+D)-D(A+B) \\
\operatorname{Img}=A(C+D)-C(A-B)\end{array}$ & $\begin{array}{l}A(C+D)=48 \\
C(A-B)=48 \\
D(A+B)=41\end{array}$ & $137 \mathrm{~mW}$ \\
\hline V & $\begin{array}{l}\text { Real }=B(C-D)+C(A-B) \\
\text { Img }=B(C-D)+D(B+A)\end{array}$ & $\begin{array}{l}\mathrm{B}(\mathrm{C}-\mathrm{D})=34 \\
\mathrm{C}(\mathrm{A}-\mathrm{B})=48 \\
\mathrm{D}(\mathrm{B}+\mathrm{A})=41\end{array}$ & $123 \mathrm{~mW}$ \\
\hline VI & $\begin{array}{l}\text { Real }=\mathrm{D}(\mathrm{A}-\mathrm{B})+\mathrm{A}(\mathrm{C}-\mathrm{D}) \\
\mathrm{Img}=\mathrm{B}(\mathrm{C}+\mathrm{D})+\mathrm{D}(\mathrm{A}-\mathrm{B})\end{array}$ & $\begin{array}{l}\mathrm{A}(\mathrm{C}-\mathrm{D})=44 \\
\mathrm{~B}(\mathrm{C}+\mathrm{D})=38 \\
\mathrm{D}(\mathrm{A}-\mathrm{B})=42\end{array}$ & $124 \mathrm{~mW}$ \\
\hline VII & $\begin{array}{l}\text { Real }=C(A+B)-B(C+D) \\
\text { Img }=C(A+B)+A(D-C)\end{array}$ & $\begin{array}{l}\mathrm{A}(\mathrm{D}-\mathrm{C})=46 \\
\mathrm{~B}(\mathrm{C}+\mathrm{D})=38 \\
\mathrm{C}(\mathrm{A}+\mathrm{B})=47\end{array}$ & $131 \mathrm{~mW}$ \\
\hline VIII & $\begin{array}{c}\text { Real }=\quad B(C-D)-C(B-A) \\
\text { Img }=C(B-A)+A(C+D)\end{array}$ & $\begin{array}{l}\mathrm{B}(\mathrm{C}-\mathrm{D})=34 \\
\mathrm{C}(\mathrm{B}-\mathrm{A})=34 \\
\mathrm{~A}(\mathrm{C}+\mathrm{D})=48\end{array}$ & $116 \mathrm{~mW}$ \\
\hline
\end{tabular}

\section{CONCLUSION}

This paper discuss about the power efficient complex multiplication scheme in FFT architecture. The pre computation in the complex multiplication allows us to generate eight different schemes. The proposed precomputation schemes have shown different dynamic power values while computing 1024 point FFT. The observations are taken over 5 data sets form MIT BIH library. The lowest power consuming scheme can be selected for the design of battery operated wearable ECG monitoring gadgets. The reason behind lower the power consumption in the specific scheme can be investigated by applying some automated techniques from data analytics. The probability of change in the values applied to the multiplication in the consecutive operations will give us the insight of efficient pre-computation method for the complex multiplication.

\section{REFERENCES}

C. Yu and M. Yen, "Area-Efficient 128- to 2048/1536Point Pipeline FFT Processor for LTE and Mobile WiMAX Systems," in IEEE Transactions on Very Large Scale Integration (VLSI) Systems, vol. 23, no. 9, pp. 17931800, Sept. 2015, doi: 10.1109/TVLSI.2014.2350017.

Fuster V.: Epidemic of Cardiovascular Disease and
Stroke: The Three Main Challenges, Circulation, Vol. 99, Issue 9, (March 1999) 1132-1137

Harland, C., Clark, T., Prance, R.: Electric Potential Probes- New Directions in the remote sensing of the human body, Measurement Science and Technology, Vol. 13, (2002) 163-169

Harland, C., Clark, T., Prance, R.: High resolution ambulatory electrocardiographic monitoring using wrist-mounted electric potential sensors, Measurement Science and Technology, Vol. 14 (2003) 923-928 https://archive.physionet.org/physiobank/database/ mitdb/

https://www.who.int/cardiovascular_diseases/about_ cvd/en/

R. Neuenfeld, M. Fonseca and E. Costa, ”Design of optimized radix-2 and radix-4 butterflies from FFT with decimation in time," 2016 IEEE 7th Latin American Symposium on Circuits \& Systems (LASCAS), Florianopolis, 2016, pp. 171-174, doi: 10.1109/ LASCAS.2016.7451037.

S. M. Noor, E. John and M. Panday, "Design and Implementation of an Ultralow-Energy FFT ASIC for Processing ECG in Cardiac Pacemakers," in IEEE 
Transactions on Very Large Scale Integration (VLSI) Systems, vol. 27, no. 4, pp. 983-987, April 2019, doi: 10.1109/TVLSI.2018.2883642.

W. Tsai, S. Chen and S. Huang, "Reconfigurable Radix- 2k×3 Feedforward FFT Architectures," 2019 IEEE International Symposium on Circuits and Systems (ISCAS), Sapporo, Japan, 2019, pp. 1-5, doi: 10.1109/ ISCAS.2019.8702346. 